\title{
A HIGH-EFFICIENCY FUSION METHOD OF MULTI-SPECTRAL IMAGE AND PANCHROMATIC IMAGE
}

\author{
Xiaorong Xue ${ }^{*}$ JiPeng Wang Hongfu Wang Fang Xiang \\ The School of Computer and Information Engineering, Anyang Normal University, Anyang, China \\ xr9986@163.com
}

KEY WORDS: Image Fusion, Multi-Spectral Image, Panchromatic Image, Parallel Computing

\begin{abstract}
:
With the development of modern remote sensing technology, a variety of earth observation satellites could continue to tremendously provide image data of different spatial resolution, time resolution, spectral resolution remote sensing, and the remote sensing data obtained is increasing with great capacity, which forms multi-source image pyramid in the same area. To play the advantages of a variety of remote sensing data, the application of remote sensing image fusion is a very important choice. When remote sensing data is large, fusion is large in computing capacity and time-consuming, so it is difficult to carry out rapid, real-time fusion. However, in some remote sensing applications, such as disaster prevention and relief quick, etc., timely fusion is required. Based on image fusion method of principal component analysis (PCA) and the advantage of parallel computing, a high-efficiency fusion method of multi-spectral image and panchromatic image is proposed. Beijing-1 Micro-satellite is a high-performance small satellite for earth observation, With Beijing-1 Micro-satellite remote sensing images as the experimental data, it is proved that good fusion results of multi-spectral image and panchromatic image can be obtained with the proposed method, and the fusion speed is also fast. At the same time, some measures of improving the efficiency of parallel image fusion are also discussed.
\end{abstract}

\section{INTRODUCTION}

With the development of modern remote sensing technology, a variety of earth observation satellites could continue to tremendously provide image data of different spatial resolution, time resolution, spectral resolution remote sensing, and the remote sensing data obtained is increasing with great capacity, which forms multi-source image pyramid in the same area. In such cases, in the description of the same object with multiple-source remote sensing information, how to conduct effective data processing, and play the advantages of a variety of remote sensing data, the application of remote sensing image fusion is a very important choice. When remote sensing data is large, fusion is large in computing capacity and time-consuming, so it is difficult to carry out rapid, real-time fusion. However, in some remote sensing applications, such as disaster monitoring, prevention and relief, dynamic monitoring on plant diseases and insect pests of agriculture, etc., rapid even real-time fusion is required[1-7].

In high-performance computing, parallel cluster computing system has a higher cost-effective ratio and good expansibility, which can meet different large-scale computing problems, and increasing importance has been attached to it. The PCA image fusion method is an important one in image fusion. In the paper, based on the analysis of the process of the PCA image fusion method, and with the advantages of parallel computing, a high-efficiency fusion method of multi-spectral image and panchromatic image is studied and proposed. Beijing-1 Micro-satellite is a high-performance small satellite for earth observation, which has multi-spectral remote sensor with the resolution of 32 meters, and panchromatic band sensor with the resolution of 4 meters(which is currently the highest resolution of small satellites in China). With Beijing-1 Micro-satellite remote sensing images as the experimental data, the experiments show that good fusion results of multi-spectral image and panchromatic image can be obtained with the proposed method, and the fusion speed is also fast. At the same

\footnotetext{
*Corresponding author. Email address: xr_986@163.com,
} flyxxr@gmail.com. time, some measures of improving the efficiency of parallel image fusion are also discussed[8-12].

\section{REMOTE SENSING IMAGE FUSION}

Multi-sensor image fusion belongs to the scope of multi-sensor information fusion, which refers to that according to a certain algorithm, the images of the same scene obtained from different sensors, or the images of the same scene obtained from the same sensor at different times are synthesized into one image which can meet certain requirement It is very difficult to simultaneously obtain high spectral, spatial and time resolution by a single sensor due to three constraints which include the resolution limit determined by the energy and diffraction of light, imaging system modulation transfer function, signal to noise ratio(SNR). Multi-sensor image fusion technology can effectively use complementary information and redundant information provided by multiple images, so the description of the scene through the image after fusion is more comprehensive and accurate than any single source image. In general, the multi-sensor information fusion technology has the following advantages: (1) It can improve system reliability and robustness. (2) It can extend the observation range of space and time.(3) It can improve the accuracy and credibility of e information. (4) It can improve performance of target monitoring and identification. (5) It can reduce the redundancy investment of the system.

Remote sensing image data fusion is divided into three levels, pixel fusion, feature fusion, decision fusion. Pixel fusion is a kind of low level integration, which is data layer integration of the raw data collected directly, and it $\mathrm{s}$ often used in multi-source images compounding, image analysis and interpretation, etc.. The process of feature level fusion is that the first step is feature extraction of the original remote sensing image information, and then comprehensive analysis and processing of the features are done so as that the fusion result can give the maximum feature information required for decision making. Decision level fusion is a high level of integration, which is the image information identification, 
classification or target detection on the basis of information provided by the pixel-level and feature-level fusion, and after obtaining information of regional decision-making, the topic image fusion is done, the integration results obtained can directly provide the basis for the command, control and decision-making system[6-7,10-14].

The research in this paper is about the pixel level fusion of Beijing-1 Micro-satellite remote sensing images.

\section{A SERIAL FUSION METHOD OF MULTI-SPECTRAL} IMAGE AND PANCHROMATIC IMAGE BASED ON PRINCIPAL COMPONENT ANALYSIS(PCA)

Principal component analysis is also known as K-L transform. It is very effective on image editing, image data compression, image enhancement, change detection, temporal dimension image fusion, etc.. The basic steps of the remote sensing image fusion based on principal component analysis (PCA) are as follows:

(1) The multi-spectral image after registration is transformed with PCA, and the first principal component PC1 is extracted;

(2) The panchromatic band image are stretched;

(3) PC1 is replaced with the stretched panchromatic band image, the inverse PCA transformation is done, and the fused image is gotten.

Image fusion based on PCA has advantages in maintaining image clarity, spectral information loss is slightly better than that of the IHS fusion method. If the follow-up application needs that the image has better spectral characteristics, PCA transform is a better choice than the IHS transformation.

The flow chart about the serial fusion method of multi-spectral image and panchromatic image Based on principal component analysis (PCA) is shown in Fig. 1:

The two Beijing-1 Micro-satellite images after registration, the panchromatic and multispectral images, are adjusted to the same image size, the three band(R, G, B) data of the multi-spectral image are input as a matrix, the size of multi-spectral image is that its height is $\mathrm{H}$, its width is $\mathrm{W}$, the size is $\mathrm{H}^{*} \mathrm{~W}$, and then the size of the input matrix IM is $3 *$ Size. The specific fusion flow chart is as the following:

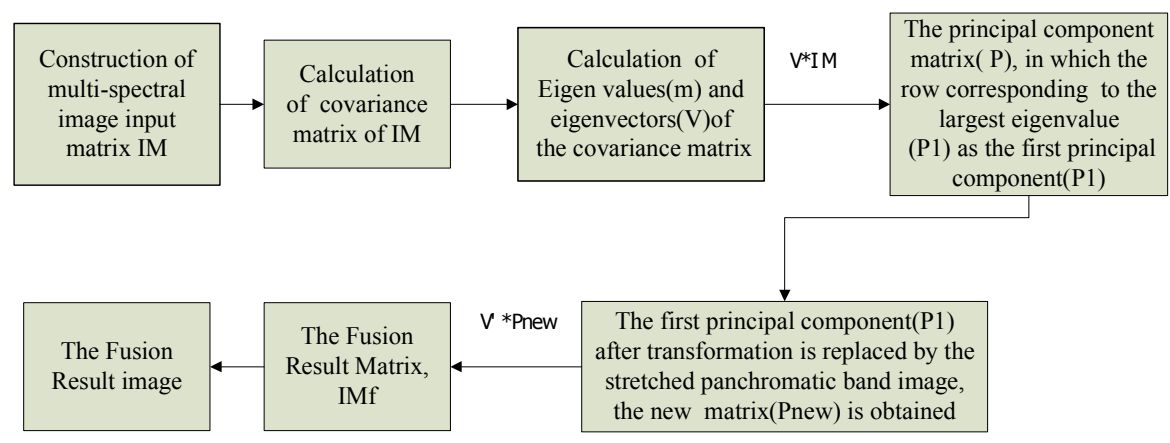

Figure 1. The flow chart about the serial fusion method of multi-spectral image and panchromatic image Based on principal component analysis (PCA)

The covariance matrix is calculated as the following, the covariance matrix can be expressed as

$$
\begin{aligned}
& {\left[\begin{array}{lll}
\sigma_{11} & \sigma_{12} & \sigma_{13} \\
\sigma_{21} & \sigma_{22} & \sigma_{23} \\
\sigma_{31} & \sigma_{32} & \sigma_{33}
\end{array}\right]} \\
& \sigma_{x y}=\operatorname{COV}(X, Y)=\frac{\sum_{i=1}^{\text {Size }}\left(X_{i}-\bar{X}\right)\left(Y_{i}-\bar{Y}\right)}{\text { Size }-1}
\end{aligned}
$$$$
(x=1,2,3 ; \quad y=1,2,3)
$$

In parallel fusion, calculation of covariance matrix elements in serial fusion can be rewritten as the following,

$X_{i}, Y_{i}$ respectively represent one of the $\mathrm{R}, \mathrm{G}, \mathrm{B}$ band value of the pixel $i$.

$$
\sigma_{x y}=\operatorname{COV}(X, Y)=\frac{\sum_{i=1}^{\text {Size }}\left(X_{i}-\bar{X}\right)\left(Y_{i}-\bar{Y}\right)}{\text { Size }-1}=\left[\sum_{i=1}^{\text {Size/n }}\left(X_{i}-\bar{X}\right)\left(Y_{i}-\bar{Y}\right)+\sum_{\text {Size/n+1 }}^{2^{*} \text { Size/n }}\left(X_{i}-\bar{X}\right)\left(Y_{i}-\bar{Y}\right)+\cdots \sum_{\text {Size* }(n-1) / n+1}^{\text {Size }}\left(X_{i}-\bar{X}\right)\left(Y_{i}-\bar{Y}\right)\right] /(\text { Size }-1)
$$

The thinking of parallel PCA fusion is as the following,

(1) According to the number of processes, image data is distributed, the global means of $\mathrm{R}, \mathrm{G}, \mathrm{B}$ component of multi-spectral image are obtained with parallel computing.

(2) According to the parallel calculation formula of the covariance matrix, (3), covariance matrix of multi-spectral image is gotten by parallel computing.
(3) Eigenvalues and eigenvectors of the covariance matrix are calculated, and eigenvectors are sorted according to the magnitude of absolute values of eigenvalues.

(4) The PCA transformation of multi-spectral image data is done with parallel computing, and the first principal component after transformation is replaced by the panchromatic band image. 
(5) Inverse PCA transform is done with parallel computing.

(6) Form the fused image.

\section{EXPERIMENT AND RESULT ANALYSIS}

In the experiments, Beijing-1 Micro-satellite remote sensing images are used for fusion.Beijing-1 Micro-satellite was successfully launched in China in 2005, it is a high-performance small satellite for earth observation, and it has advanced performance, short development cycle and low cost. The satellite is a small satellite for earth observation with two remote sensors of medium and high resolution. The medium resolution remote sensor is multi-spectral with 32 meters resolution, the high-resolution remote sensor is panchromatic remote sensor with 4 meters resolution. The 4 meters panchromatic band sensor of Beijing-1 micro-satellite is currently the highest resolution of small satellites in China. The spectrum range of the multi-spectral sensor with 32-meter resolution includes green, red, and near infrared band. In promoting economic development and social coordination, it can make full use of satellite's advantages of remote sensing monitoring such as great range, high speed, data reliability, etc.. Beijing-1 Micro-satellite can provide a large amount of remote sensing data with high-resolution. To play its role better, it is necessary to combine the respective merits of its multi-spectral image data and panchromatic data, or the satellite remote sensing data with other remote sensing data, and it is very important to explore the rapid image fusion technology for playing the role of Beijing-1 Micro-satellite well, which has an important theoretical value and a wide range of applications[11-12].

The images obtained from image data distribution department are their two level products, which are the standard image data through the system radiometric calibration, system geometric correction. After the process, the three-band multi-spectral image and panchromatic image in the same area obtained are used as the basis data for image fusion.

We achieve the parallel algorithm on the parallel cluster system. Because the size of the image affects the parallel performance of the algorithm, in the experiments, we use different sizes of the Beijing-1 Micro-satellite images and do experiment with different number of processes, and do analysis and comparison of the results. The test platform is configured as the following, 8 computers constitute a cluster architecture (one as the management node (also do computing ), 7 computing nodes), each node is configured as the following, its CPU is Intel(R) Core(TM)2 Duo CPU, memory is $2 \mathrm{G}$, the 8 computers are interconnected through the 100Mbps Fast Ethernet switch, and the software environment is made up of the WindowsXP operating system, NT-MPICH message passing parallel libraries, etc.. Figure. 2 and Figure.3, Figure. 5 and Figure .6 are the thumbnails for the two groups of original panchromatic image and multispectral image of Beijing-1 Micro-satellite. Figure. 4 and Figure. 7 are the thumbnails for the corresponding fusion results.

To test the algorithm's parallel performance, some images of Beijing-1 Micro-satellite with different size are used to test on different number of computers. Table 1 gives the cost time and parallel efficiency of fusion for images of Beijing-1 Micro-satellite with different size on 1,4,8 and 16 computers respectively. The experiment results show that good result of fusion can be obtained quickly with the new method. On the other hand, the algorithm's parallel performance is affected by the time of data transfer, the time can be reduced by improving the network bandwidth. By the analysis of the relation between the efficiency of the new method and network bandwidth, it is discovered that the network bandwidth has effect on the efficiency, the wider the network bandwidth, the higher the efficiency, and the better the extensible function.

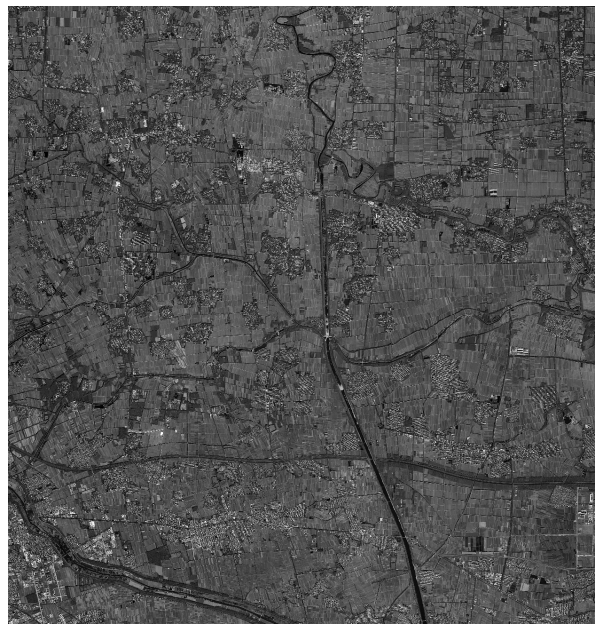

Figure 2. The panchromatic image of Beijing-1 small satellite(P1)

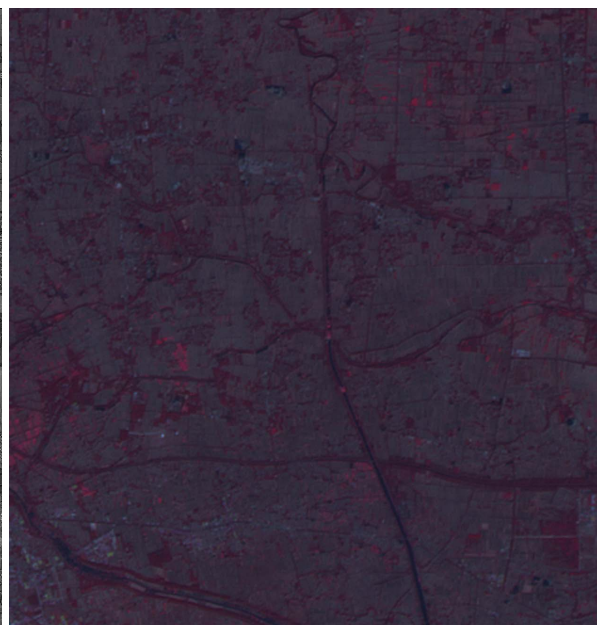

Figure 3. The multispectral image of Beijing-1 small satellite(M1)

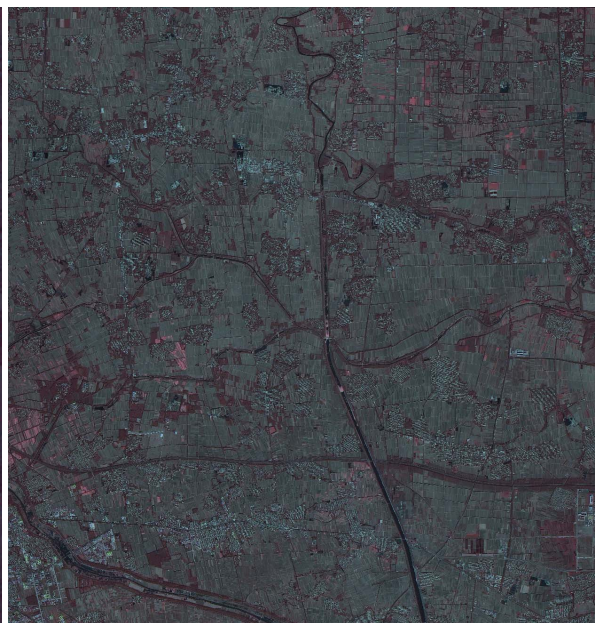

Figure 4.The fusion result image of Beijing-1 small satellite with the proposed method of image fusion in the paper(F1) 


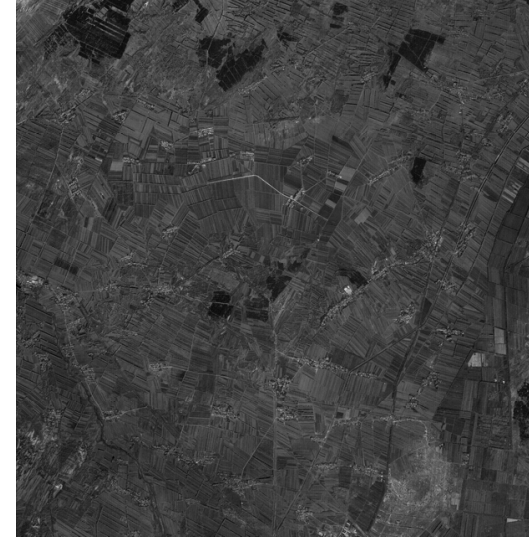

Figure 5. The panchromatic image of Beijing-1 small satellite(P2)

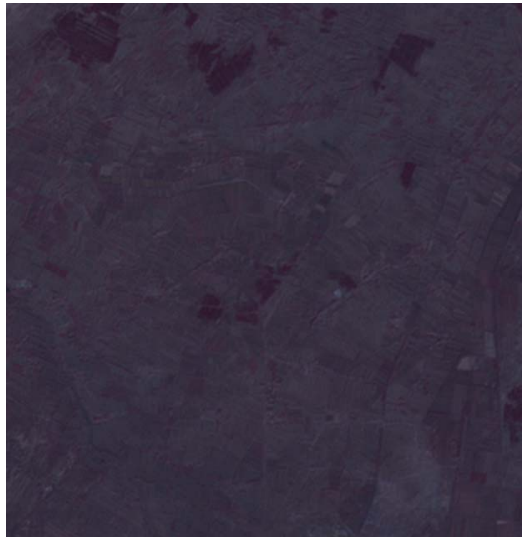

Figure 6. The multispectral image of Beijing-1 small satellite(M2)

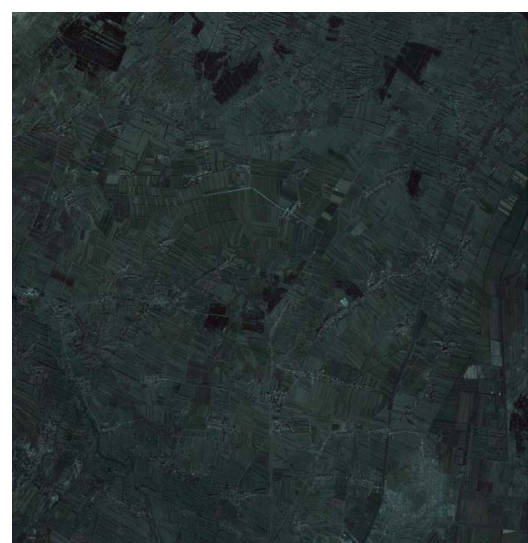

Figure 7.The fusion result image of Beijing-1 small satellite with the proposed method of image fusion in the paper(F2)

\begin{tabular}{|c|c|c|c|c|c|}
\hline \multicolumn{2}{|c|}{ Number of computers } & 1 & 2 & 4 & 8 \\
\hline \multirow{2}{*}{$\begin{array}{c}\text { Fusion one } \\
\text { (Figure. 5-7) }\end{array}$} & Time cost (ms) & 31125 & 15905 & 9836 & 6256 \\
\cline { 2 - 5 } & Efficiency & & 0.511 & 0.316 & 0.201 \\
\hline $\begin{array}{c}\text { Fusion two } \\
\text { (Figure. 2-3) }\end{array}$ & Time cost (ms) & 166812 & 84073 & 51879 & 32528 \\
\cline { 2 - 6 } & Efficiency & & 0.504 & 0.311 & 0.195 \\
\hline
\end{tabular}

Table 1. The parallel efficiency(Efficiency $=$ running time on $\mathrm{n}$ computers/ running time on one computer) 2007.

\section{CONCLUSION}

In order to play the advantages of a variety of remote sensing data for earth observation, based on the principal component analysis(PCA) image fusion method and parallel computing, a high-efficiency fusion method of multi-spectral image and panchromatic image is studied and realized in the environment of distributed memory. In the paper, the algorithm is discussed, and it is achieved on a cluster system. The parallel fusion method is tested with different sizes of multispectral and panchromatic images of Beijing-1 Micro-satellite. The results show that the new algorithm has better convergence results and good parallel performance, and the wider the network bandwidth, the higher the efficiency, and the better the extensible function.

\section{ACKNOWLEDGEMENT}

The paper is supported by (1) the National Natural Science Foundation Project of China (U1204402),(2) the Foundation Project for Innovation(supported by Digital China Research Institute of Peking University and the twenty-first century Aerospace technology Co., Ltd., China), (3) the Science and Technology project(122102210462) supported by the Department of Science and Technology in Henan province, China,(4) the Fund project for young teachers of universities in Henan province, China, (5) Science and Technology Development Project of Anyang (117), China.

\section{REFERENCES}

[1] Kang Yao-hong. Data Fusion Theory and Applications(The Second Edition) [M]. Xi'an: Xidian University Press, 2006.

[2] Jing Zhong-Liang. Image Fusion -Theory and Applications [M]. Beijing: Higher Education Press(China),
[3] Henrik Aanæs, Johannes R. Sveinsson, Allan Aasbjerg Nielsen,Thomas Bøvith, and Jón Atli Benediktsson.Model-Based Satellite Image Fusion,2008, IEEE Transactions on Geoscience and Remote Sensing,46(5):1336-1346.

[4] Jaewan Choi, Kiyun Yu, and Yongil Kim.A New Adaptive Component-Substitution-Based Satellite Image Fusion by Using Partial Replacement, IEEE Transactions on Geoscience and Remote Sensing,2011,49(1):295-309.

[5] Sheng Zheng, Wen-zhong Shi, Jian Liu. Remote Sensing Image Fusion Using Multiscale Mapped LS-SVM, IEEE Transactions on Geoscience and Remote Sensing, 2008,46(5):1313-1322.

[6] Arash Golibagh Mahyari and Mehran Yazdi.Panchromatic and Multispectral Image Fusion Based on Maximization of Both Spectral and Spatial Similarities, IEEE Transactions on Geoscience and Remote Sensing,2011,49(6):1976-1985.

[7] Myungjin Choi.A New Intensity-Hue-Saturation Fusion Approach to Image Fusion With a Tradeoff Parameter, IEEE Transactions on Geoscience and Remote Sensing, $2006,44(6): 1672-1682$.

[8] Chen Guoliang, An Hong. Practice of Parallel Algorithms [M]. Beijing: Higher Education Press, 2004.

[9] Du Zhihui. Parallel Programming of High Performance Computing ---MPI parallel program design [M]. Beijing: Tsinghua University Press, 2001.

[10] Hu Xiaodong, Luo Jiancheng, Shen Zhanfeng, WU Wei, CHEN Qiuxiao.Data sewing algorithm for parallel segmentation of high-resolution remotely sensed image, Journal of Remote Sensing, 2010,14(5): 917-927.

[11] Tong Qingxi, Wei Zheng. Beijing-1 Micro-satellite and its data applications, spacecraft engineering, 2007,16 (2) :1-5.

[12] Zeng Tao, Liu Jianqiang. The Application of Beijing Micro -Satellite Data to Algae Disaster Monitoring in the Sea of Qingdao, Application of Remote Sensing, 2009,No.3: 34 -37 . 